\title{
ANALYSIS OF MECHANICAL ARM CONTROL METHODS UNDER NON-LINEAR DIFFERENTIAL EQUATION
}

\author{
Haiming Fu ${ }^{1}$, Guiping Liang ${ }^{1}$, Quoc Tien Le $e^{2}$ \\ ${ }^{1}$ Guangzhou Huaxia Vocational College, Guangzhou Guangdong 510935, China \\ ${ }^{2}$ Haiphong Department of Education and Training, Haiphong, Vietnam, \\ Email: gpliang935@163.com
}

\begin{abstract}
In order to track the trajectory of the mechanical arm in realtime, the radial basis function neural network algorithm is used to optimize the control system of the mechanical arm. Firstly, the neural network and radial basis neural network algorithm are briefly introduced. Then, the controller of the mechanical arm is designed using radial basis function neural network algorithm, and the control method of the mechanical arm under ideal model and the mechanical arm control method with interference and uncertainty are designed according to the operation state of the mechanical arm. Finally, the control methods of the mechanical arm under ideal model mechanical arm under interference and uncertainty are simulated. The radial basis function neural network algorithm proposed in this research has strong self-learning characteristics, parallel processing ability, and nonlinear mapping ability.Moreover, the network weight can be adjusted online adaptively under the precise dynamic model without the need of mechanical arm to compensate for the gravity; when there are uncertain gravity terms in mechanical arm control system. The algorithm based on radial basis function neural network proposed in this research has better tracking speed and accuracy. This research has promoted the development of robot industry.
\end{abstract}

Keywords: Nonlinear Differential Equations, Mechanical Arm, Control Method, Radial Basis Function Neural Network Algorithm.

\section{Introduction}

With the emergence of science and technology, the robot invented by human has attracted the attention of the whole world. From the middle of the 20th century to the present, the research on robots has not stopped, and many achievements have been made, making the application range of robots very diversified. The application of robots has penetrated from the traditional industrial manufacturing field to the military field, aerospace, service industry, medical industry, and every aspect of daily human life. Robots have become a good helper of daily human life, which has significantly improved industrial efficiency and people's daily life quality [1].

After decades of research, robots have evolved rapidly, and now robots are strictly related to human life. However, the robots currently built do not have a human mind and can only operate according to the instructions set by humans. For mechanical arm control, a variety of control methods have been emerged [2]. Among these control methods, the Proportion Integration Differentiation (PID) control system is widely used in the robot's mechanical arm control system. Although PID control system has been very mature, but as the system gradually becomes complex, the dynamic performance of PID control system is not good. Besides, there are also some minor flaws in the calculation of torque control and robust control, but it shows good performance in adjusting time and overshooting [3]. For the control system of the mechanical arm, the common disadvantage is that it depends on the parameters of the mathematical model of the mechanical arm. They all require a mechanical arm with a precise mathematical model or give the uncertainty of the system boundary. However, in the case of time, incomplete information, no precisely defined parameters, and external interference factors (such as the control method of sensor information) appear during the operation of the mechanical arm. Moreover, it has a high sensitivity to accidental or unplanned times. As a result, the traditional modelbased robot control method can't guarantee the robustness of the system in the actual unpredictable environment [4-5]. Therefore, it is necessary to find a new hybrid control method to solve similar problems. Since neural network algorithm has good self-learning characteristics, parallel processing ability, nonlinear mapping ability, and good fault tolerance, it is widely used in mechanical arm control. Therefore, the neural network algorithm is combined with other control methods to design the controller to meet the requirements of the mechanical arm in track.

To sum up, in order to understand the tracking effect of a nonlinear strongly coupled mechanical 
arm with high precision and speed, the algorithm based on Radial Function Basis (RBF) neural network is proposed to realize the control of the mechanical arm. Firstly, the neural network algorithm and the radial basis function neural network algorithm are introduced. Then, according to the running state of the mechanical arm, the control method of the mechanical arm under the ideal model and the mechanical arm control algorithm with interference and uncertainties are designed. Finally, simulation experiments are carried out for the control methods of mechanical arm under two different conditions. It is expected that the research can provide good guidance for the control method of a mechanical arm.

\section{Methodology \\ 2.1 The neural network}

An artificial neural network is a large-scale nonlinear adaptive system. The theory of human brain activity can be formed into a mathematical model with the characteristics of self-learning ability, nonlinear approximation ability, and parallel distribution. At present, the application of artificial neural network in mechanical arm control system is widespread [6-8].

An artificial neuron is the most basic information processing unit of a neural network, as well as the premise and basis of neural network to ensure the normal operation of neural network. The structure of the artificial neural network be an analog adder, and its input signals can be seen as continuous variables or discrete pulses. When each input signal is transmitted, it produces a weight, which can also be a connection strength, like a biological neuron.

When the positive weight is generated, it will stimulate the mechanism; when the weight value is negative, it will inhibit the mechanism. In an artificial neural network, linear function is the simplest excitation function, but the most common excitation function is nonlinear. Therefore, in the nonlinear excitation function, neurons need to realize off-site input and output mapping [9-10].

The advantages of neural network are as follows:

- A small amount of prior knowledge can be used for self-learning. This property is liberation from complex mathematical computation and can play a powerful role in the control field involving theoretical computation. The general control algorithm needs a lot of model prior information to design the control scheme, while the controller does not need too much system model and parameter information. Therefore, neural network control can be widely used to solve the control problem of uncertain models [11].

- Since some single control methods are powerless to the nonlinear system, the nonlinear function in the neural network algorithm is used to realize the nonlinear mapping function [12-13].

- The distributed structure and parallel computing methods of the neural network make it faulttolerant. Some connection errors have little impact on the system as a whole [14-15].

\subsection{RBF neural network algorithm}

RBF neural network algorithm is a three-layer forward network. Its network topology can be described as following: firstly, through training data set, mainly through Affinity Propagation (AP) clustering, the hidden layer and node center can be further obtained; then, the genetic algorithm is used to optimize the global searching ability to find the optimal radial basis function and connection weight; finally, a second search is conducted, mainly using chaos search to search the optimal individuals and differences of the population [16].

The convergence analysis based on radial neural network algorithm means that the probability of the scaling factor $F$ can be converged at 1 by using genetic algorithm under the condition that the scaling factor $\mathrm{F}$ is certain. Assuming that the genetic algorithm converges to the global optimal solution $\mathrm{X}_{\text {best, }}$ then individual $\mathrm{X}_{\mathrm{i}}(\mathrm{t})$ of the $\mathrm{t}$ th iteration can be expressed as Eq. 1.

$$
\lim _{t \rightarrow \infty} X_{i}(t)=X_{i}^{\text {best }}, i \in(1,2, \cdots, n)
$$

Eq. 1 can be converged using RBF algorithm, and Eq. 2 is solved by the stability determination method of the nonlinear equilibrium point.

$$
z=f(\mathrm{z})=\mu z(1-\mathrm{z})
$$

Because the radial basis function needs to compete according to the fitness function, the solution of this equation can only be one of the convergent values, which proves that the radial basis function has convergence.

The approximation theorem for RBF neural network algorithm was proposed in the late 1980s. Assuming that there are some function $f\left(x_{1}, x_{2}, \mathrm{~L}, x_{n}\right)$ and the accuracy is greater than zero and less than some constant, then, there is a forward network whose implicit function is $\sigma(x)$. Besides, there is a linear relationship between the input layer and the output layer. The relationship between the total input and the output of the neural network can be expressed as follows.

$$
Y=\hat{f}\left(x_{1}, x_{2}, \cdots, x_{n}\right)
$$

Because RBF neural network is also a multi-layer forward network, which also satisfies the approximation theorem, therefore, an arbitrary approximation can be used to define nonlinear functions over R in single-layer RBF networks.

By combining the traditional control method with the on-line adaptive RBF neural network control method, the stability of the system is proved by Lyapunov analysis method. It is very effective to use 
RBF neural network algorithm to make an adaptive approximation to unknown parts.

Assuming that there's an estimate function $f(x)$, RBF neural network algorithm can be used to obtain Eq. 4 and Eq. 5.

$$
\begin{aligned}
& h_{j}=\exp \left(\phi-c_{i j} \|^{2} / b_{j}^{2}\right) \\
& f=W^{* T} h(x)+\eta
\end{aligned}
$$

In Eq. 5, $\eta$ is the approximation error.
If the network input is taken as the state of the system, then the network output can be expressed as follows.

$$
\hat{f}(x)=W^{* T} h(x) h(x)
$$

\subsection{Ideal model control method}

For the mechanical with $\mathrm{n}$ connecting rod, its dynamic equation is expressed as follows.

$$
M(q) \ddot{q}+C(q, \dot{q}) \dot{q}+G(q)+F(\dot{q})+\tau_{d}(q, \dot{q}, t)=\tau
$$

In Eq. $7, F$ represents the friction matrix; $\tau_{d}(q, \dot{q}, t)$ represents external interference and unmodeled dynamic vector; $\int_{0}^{\infty} \tau_{d}(q, \dot{q}, t) \mathrm{i}$ is definite.

If the friction force is ignored, then the dynamic equation expression is as follows.

$$
M(q) \ddot{q}+C(q, \dot{q}) \dot{q}+G(q)+\tau_{d}=\tau
$$

If there is no external interference, the PD control expression based on gravity compensation is as follows.

$$
\tau=K_{p} e+K_{d} \dot{e}+\widehat{G}(q)
$$

In Eq. 9, $\hat{G}(q)$ represents the estimated value of the moment of gravity; $K_{p}$ and $K_{d}$ are expressedas positive definite matrixes.

The tracking error of this research is as follows.

$e=q_{d}-q$

When fixed-point control is adopted, $q_{d}$ is a constant value, then Eq. 11 is obtained.

$$
\dot{q}_{d}=\ddot{q}_{d}
$$

Therefore, the equation of the mechanical arm can be expressed as follows.

$$
M(q)\left(\ddot{q}_{d}-\ddot{q}\right)+C(q, \dot{q})\left(\dot{q}_{d}-\dot{q}\right)+K_{p} e+K_{d} \dot{e}+\hat{G}(q)-G(q)=\tau
$$

From the expression of PD control law, it can be concluded that the correct evaluation of the heavy torque is needed to realize the control law.

Because when the estimate of the moment of gravity is correct, the below equation exists.

$$
\widehat{G}(q)=G(q)
$$

Then Eq. 14 is obtained.

$$
M(q) \ddot{e}+C(q, \dot{q}) \dot{e}+K_{P} e=-K_{p} e
$$

After designing the controller, stability analysis is needed. Eq. 15 is obtained according to the Lyapunov function.

$$
L=\dot{e}^{T} M \ddot{e}+\dot{e}^{T} C \dot{e}+\dot{e}^{T} K_{p} e=\dot{e}^{T}(M \ddot{e}+C \dot{e}+e)=-\dot{e}^{T} K_{p} K_{p} \leq 0
$$

Because of the fact that $\dot{L}$ is semi-negative, and $K_{d}$ means positive definite. Therefore, when $L \equiv 0$, then $\dot{e} \equiv 0$ and $\ddot{e} \equiv 0$ is obtained. By substituting $\dot{e}$ and $\ddot{e}$ into Eq. 12, $K_{p} e \equiv 0$ is obtained. According to the reversibility of $K_{p}, e \equiv 0$ is obtained.

According to LaSalle theorem, $(e, \dot{e})=(0,0)$ is the asymptotically stable equilibrium point of the mechanical arm, that is, when $t \rightarrow \infty$, then there is $q \rightarrow q_{d}, \dot{q} \rightarrow q_{d}$

$$
L=\frac{1}{2} \dot{e}^{T} M(q) \dot{e}+\frac{1}{2} e^{T} K_{p} e
$$

According to the positive qualitative of $M(q)$ and $K_{p}$, L is a global positive definite, and then the derivative is taken of it.

$$
L=\frac{1}{2} \dot{e}^{T} M(q) \dot{e}+\frac{1}{2} e^{T} K_{p} e+\dot{e}^{T} K_{p} e
$$

According to the characteristics of the mechanical arm, there's a symmetric property for $\dot{M}-2 C$.

$$
\dot{e}^{T} \dot{M} \dot{e}=2 \dot{e}^{T} C \dot{e}
$$

Eq. 18 is obtained by substituting Eq. 8 into Eq. 10.

\subsection{Control methods with interference and uncertainties}

Because the mechanical arm system, in reality, can't reach the ideal state of the working environment, that is, there is no mechanical arm system without external interference. On this basis, the uncertainty of gravity term and the existence of external disturbance of the system are considered to design the control system of the mechanical arm. 
For RBF neural network approximation, two constraints are defined in this research.

I. The output of the neural network $\hat{f}(x, \omega)$ is continuous;

II. $\hat{f}\left(x, \omega^{*}\right)$ represents the ideal approximation function, then there's a smaller normal number $\varepsilon_{0}$ that satisfies Eq. 19.

$$
\max \left\|\hat{f}\left(x, \varpi^{*}\right)-f(x)\right\| \leq \varepsilon_{0}
$$

In Eq. $19, \omega^{*}$ represents the order matrix and neural network weights.

Assume that $\eta$ is the error of the approximate optimal state, which can be expressed as Eq. 20.

$$
\eta=\hat{f}\left(x, \varpi^{*}\right)-f(x)
$$

Because in the approximation ability of RBF neural network, $\eta$ is definite. Then it is set as $\eta_{0}$ and Eq. 21 is obtained.

$$
\eta_{0}=\sup \left\|\hat{f}\left(x, \varpi^{*}\right)-f(x)\right\|
$$

Among them, Eq. 22 is expressed as follows.

$$
\hat{f}\left(x, \varpi^{*}\right)=\varpi^{* T} h(x)
$$

In this research, RBF neural network is used to approximate the gravity term, then. $f(x)=G(q)$

In the controller design of RBF neural network method, the new control law is as fflbows.

$$
\tau=K_{p} e+K_{d} \dot{e}+\hat{f}(x, \varpi)
$$

$v=\left(\eta_{0}+b_{d}\right) \operatorname{sgn}(\dot{e})$ represents a robust term that cancels out the error from the neural network approximation. $\operatorname{sgn}(\dot{e})$ is expressed as follows.

$$
\operatorname{sgn}(\dot{e})\left\{\begin{array}{c}
1, \dot{e}>0 \\
0, \dot{e}=0 \\
-1, \dot{e}<0
\end{array}\right.
$$

Thus, the mechanical arm equation can be changed to Eq. 25.

$$
M(q)\left(\ddot{q}_{d}-\ddot{q}\right)+C(q, \dot{q})\left(\ddot{q}_{d}-\ddot{q}\right)+K_{p} e+K_{d} \dot{e}+\hat{f}\left(x, \varpi^{*}\right)-f(x)-\tau_{d}-v=0
$$

Among them, Eq. 26 and Eq. 27 are expressed as follows.

$$
\begin{aligned}
& \quad \hat{f}\left(x, \varpi^{*}\right)-f(x)=\hat{f}(x, \varpi)-f\left(x, \varpi^{*}\right)-f(x)=\widehat{\varpi}^{T} h(x)-\varpi^{*} h(x)+\eta=-\widehat{\varpi}^{T} h(x) \\
& \widetilde{\varpi}=\varpi^{*}-\varpi \quad \widetilde{\varpi}=-F h(x) \dot{e}^{T}
\end{aligned}
$$

Therefore, the adaptive law of the design is as follows.

According to the Lyapunov function, Eq. 29 is obtained.

$$
L=\frac{1}{2} \dot{e}^{T} M(q) \dot{e}+\frac{1}{2} e^{T} K_{p} e+\frac{1}{2} \operatorname{tr}\left(\widetilde{\varpi}^{T} F^{-1} \widetilde{\varpi}\right)
$$

In Eq. 29, $\mathrm{F}$ represents the symmetric positive definite matrix, and $\mathrm{L}$ represents the global positive

definite.

Eq. 30 is obtained by taking derivation of Eq. 23.

$$
L=\dot{e}^{T} M \ddot{e}+\frac{1}{2} \dot{e}^{T} C \ddot{e}+\dot{e}^{T} K_{p} e+\operatorname{tr}\left(\widetilde{\varpi}^{T} F^{-1} \dot{\widetilde{\varpi}}\right)
$$

According to the characteristics of the mechanical

expressed as follows. arm, $\quad \dot{e}^{T} \dot{M} \dot{e}=2 \dot{e}^{T} C \dot{e}$ is obtained, then Eq. 30 is

$$
L=\dot{e}^{T} M \ddot{e}+\dot{e}^{T} C \ddot{e}+\dot{e}^{T} K_{p} e+\operatorname{tr}\left(\widetilde{\varpi}^{T} F^{-1} \dot{\widetilde{\varpi}}\right)
$$

By substituting the mechanical arm obtained.

$\dot{e}^{T} \dot{M} \dot{e}=2 \dot{e}^{T} C \dot{e}$ equation into Eq. 25, Eq. 32 is

$$
\begin{aligned}
& \dot{L}=\dot{e}^{T}\left(-K_{d} \dot{e}-\widetilde{\omega}^{T}+\eta+\tau_{d}+v\right)+\operatorname{tr}\left(\widetilde{\omega}^{T} F^{-1} \dot{\tilde{\omega}}\right) \\
& =-\dot{e}^{T} K_{d} \dot{e}-\dot{e}^{T} \widetilde{\omega}^{T} h+\operatorname{tr}\left(\widetilde{\omega}^{T} F^{-1} \dot{\tilde{\omega}}\right)+\dot{e}^{T}\left(\eta+\tau_{d}+v\right) \\
& =-\dot{e}^{T} K_{d} \dot{e}+\operatorname{tr} \widetilde{\omega}^{T}\left(F^{-1} \dot{\tilde{\omega}}-h \dot{e}^{T}\right)+\dot{e}^{T}\left(\eta+\tau_{d}+v\right)
\end{aligned}
$$

There are equations as Eq. 33 and Eq. 34.

$$
\dot{\widetilde{\varpi}}=\dot{\varpi}^{*}-\dot{\hat{\varpi}}=-\dot{\hat{\sigma}}=F h(x) \dot{e}^{T}
$$

$$
\dot{e}^{T}\left(\eta+\tau_{d}+v\right)=\dot{e}^{T}\left(\eta+\tau_{d}\right)+\dot{e}^{T} v=\dot{e}^{T}\left(\eta+\tau_{d}\right)-\|\dot{e}\|\left(\eta_{0}+b_{d}\right)
$$

Then Eq. 35 is obtained.

$$
\dot{L}=-\dot{e}^{T} K_{d} \dot{e}+\dot{e}^{T}\left(\eta+\tau_{d}+v\right) \leq 0
$$

Through mathematical analysis, it can be concluded that the equilibrium state of the system is uniformly stable. Therefore, simulation is conducted 
to prove its stability, and the control effect is compared with the simulation results.

\section{Results and Discussion}

\subsection{Simulation results analysis of the ideal model control method}

In this research, a mechanical arm system with two degrees of freedom is selected for the simulation experiment. The mass of the first joint of the mechanical arm is $2.04 \mathrm{~kg}$, and the length of the first joint is $1 \mathrm{~m}$. The mass of the second joint is $1 \mathrm{~kg}$, and the length of the second joint is $0.86 \mathrm{~m}$.

According to the calculation, the initial position state of the two joints are taken respectively $q_{1}(0)=q_{2}(0)=0$ the initial velocity state is $\dot{q}_{1}(0)=\dot{q}_{2}(0)=0$. The ideal position of the two joint mechanical arm is 1 and 0.5 , respectively.

The joint parameter is,

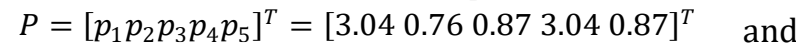
controller parameter is $K_{P}=\operatorname{diag}\{150,150\}, K_{d}=\operatorname{diag}\{70,70\}$. Figure 1 is the position tracking simulation diagram of the two joints. The ideal model control method is adopted to track the position of the two joints of the mechanical arm, and the simulation results are outstanding. At the same time, it also shows that the mechanical arm can be controlled by PD control. Figure 2 shows the output torques of the two joints.

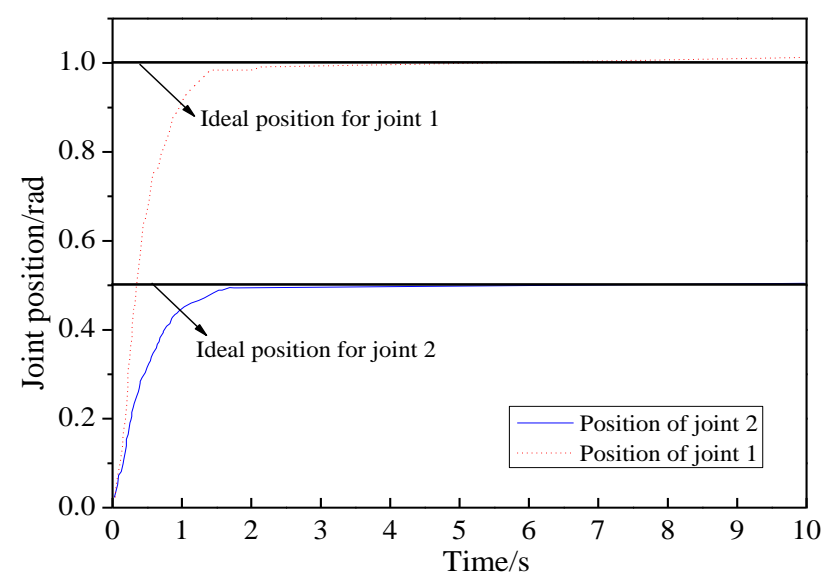

Figure 1: The position tracking of the two joints.

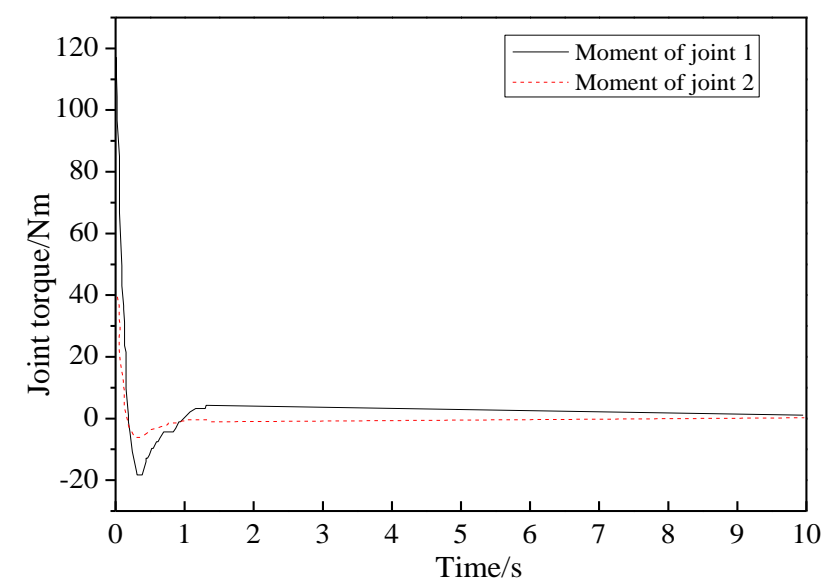

Figure 2: The output torque of the two joints.

\subsection{The simulation results analysis of the control method with disturbance and uncertainty}

Similarly, in order to verify the effectiveness of the mechanical arm control method in the presence of interference and uncertainty, the same mechanical arm model as in section 3.1 is adopted.

According to the calculation, the initial position state of the two joints are taken respectively $q_{1}(0)=q_{2}(0)=0$, the initial velocity state is $\dot{q}_{1}(0)=\dot{q}_{2}(0)=0$. The ideal position of the twojoint mechanical arm is 1.5 and 3 , respectively. RBF neural network input is joint position and joint speed, and controller parameters are selected as: $K_{p}=\operatorname{diag}\{150,150\}, K_{d}=\operatorname{diag}\{70,70\}$, $F=\operatorname{diag}\{10,10\}$, the robust item $\eta_{0}=0.3, b_{d}=0.2$. The gaussian parameter is $C=\left(\begin{array}{cccc}1 & 1 & 1 & 1 \\ 0.01 & 0.01 & 0.01 & 0.01\end{array}\right), \quad b=0.53$, and the RBF 
network topology is 2-5-1. In order to verify the superiority of the proposed algorithm, the traditional PID control, PD control with fixed gravity compensation, and PD control with adaptive RBF neural network are compared.

Figure 3 is the position tracking simulation diagram of joint 1 . The traditional PID control can overshoot the position curve by $8.3 \%$. The significant overshoot of the position curve will have a particular influence on the operation of the mechanical arm, which will affect the stable operation of the mechanical arm. While the adjustment time of fixed gravity compensation PD control is $5.2 \mathrm{~s}$, it can be concluded from the figure that it takes the longest time to reach the stable state among the three methods; the RBF neural network control method based on gravity compensation has no curve overshot, and the time needed to reach the stable running state is the shortest. Similarly, in joint 2, overshoot also occurs using traditional PID control. However, the adjustment time of fixed gravity compensation proportional differential (PD) control only takes $4.3 \mathrm{~s}$. In contrast, the RBF neural network control method based on gravity compensation needs $2 \mathrm{~s}$ to reach stable operation state, which indicates that the RBF neural network control method based on gravity compensation shows good dynamic performance, as shown in Figure 4.

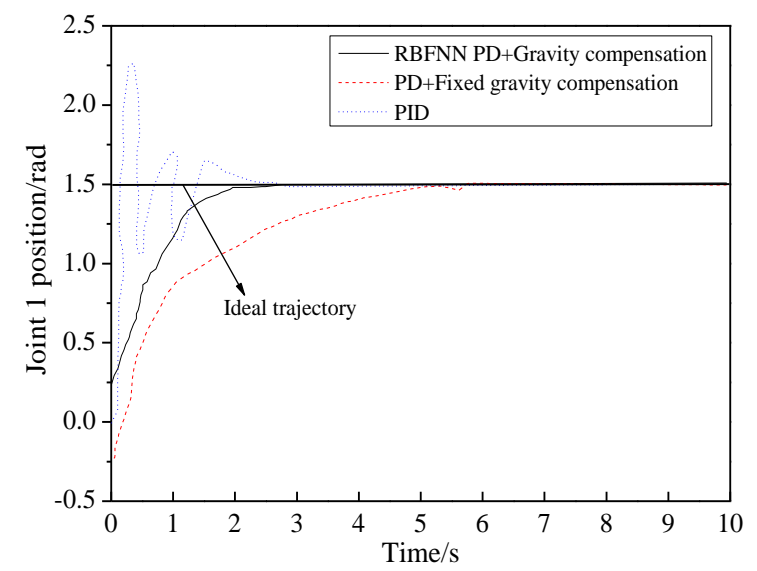

Figure 3: The position tracking curve of joint 1.

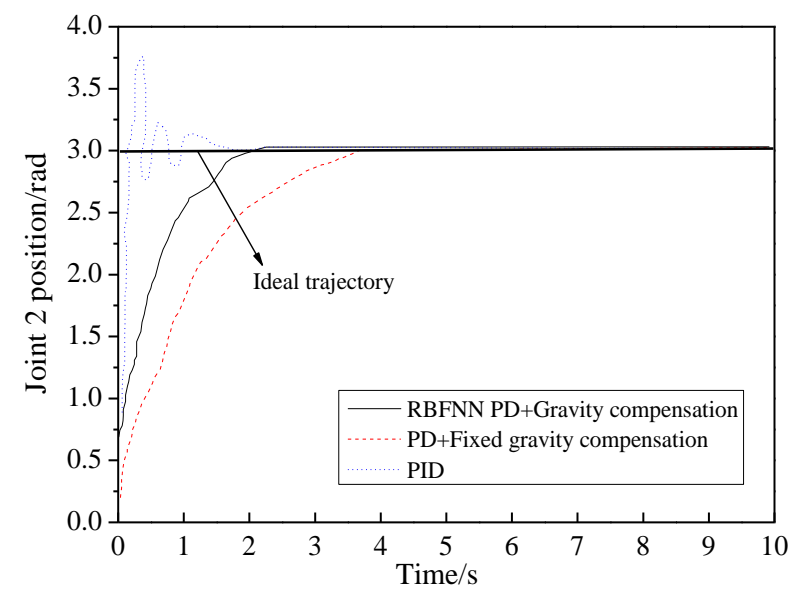

Figure 4: The position tracking curve of joint two.
Figure 5 shows the error curves of two joints of two mechanical arm. The error of stable state of the two joints is minimized by RBF neural network control method based on gravity compensation.

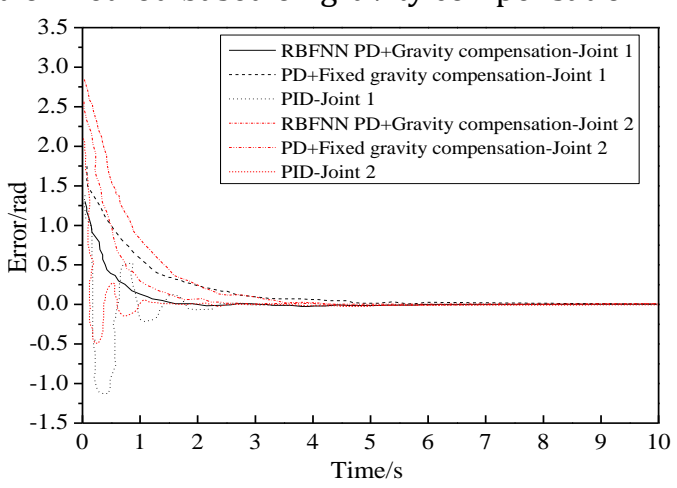

Figure 5: Tracking error of two joints.

Figure 6 and Figure 7, respectively show the output torques of joint 1 and joint 2 under three different methods. It can be concluded from Figure 6 and Figure 7 that the RBF neural network control method based on gravity compensation in the two joints has good smoothness in the output torque, followed by the RBF neural network control method, and finally the PID control method. In joint 1 and 2 , the initial torque of PID control method fluctuates greatly. In the actual production, PID control has a high requirement on servo motor, which will also increase the burden of hardware equipment.

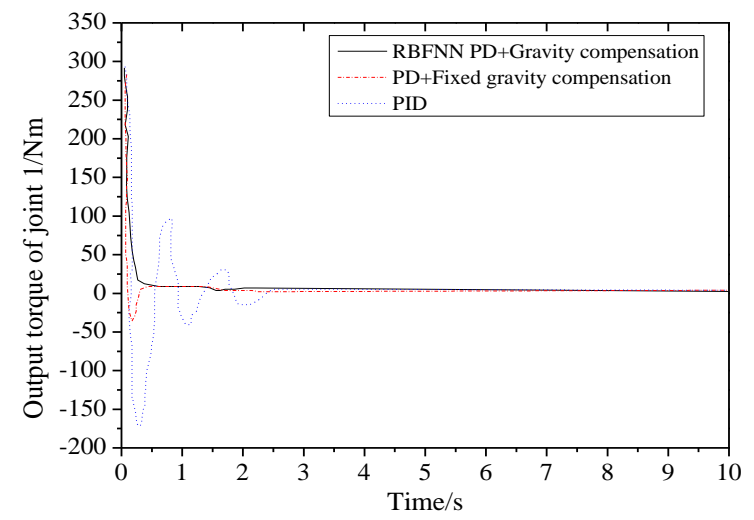

Figure 6: The output torque of joint 1.

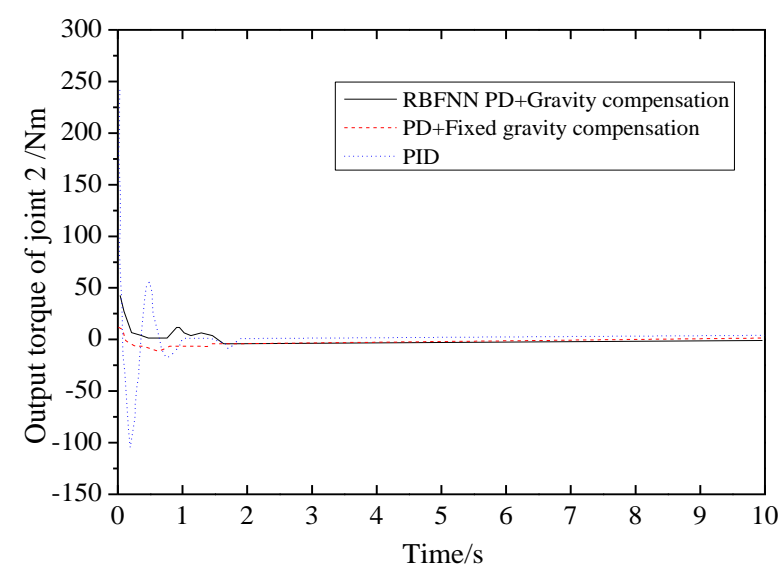

Figure 7: The output torque of joint 2. 
Figure. 8 shows the approximate effect diagram of RBF neural network based on gravity compensation on gravity term. PD control accuracy in fixed gravity compensation is relatively poor, and it takes a long time to reach the stable state, but in the initial phase the output torque is smooth. This ensures that the output torque of the PID controller in the initial stage is the maximum. Therefore, the RBF neural network controller based on gravity compensation proposed in this research has higher accuracy in tracking of the mechanical arm and a better overall control effect on the mechanical arm.

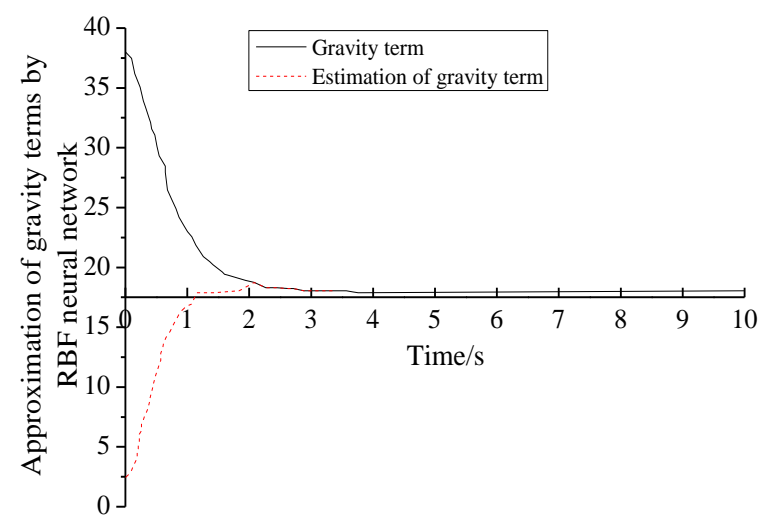

Figure 8: Approximation of gravity term by $R B F$ neural network.

\section{Conclusion}

The research aims to improve the tracking speed andaccuracy of the mechanical arm. Because radial basis function has good ability of nonlinear mapping, self-learning, and parallel processing, radial basis function neural network algorithm is used to optimize the mechanical arm control method. Firstly, the neural network algorithm and radial basis function neural network algorithm are introduced. Then, according to the running state of the mechanical arm, the control method based on the ideal model and the radial basis function neural network algorithm with disturbance and uncertainty are designed. Finally, the simulation results of the control methods under two conditions are analysed. Based on the control method under the ideal model, the RBF neural network control method based on gravity compensation is proposed. The simulation results show that the tracking speed and tracking accuracy of the mechanical arm are significantly improved by the radial basis function based neural network algorithm when the gravity term is uncertain.

This study provides a good idea for the control method of the robot mechanical arm in China.
Although good research results have been obtained in this research, since the initial velocity and acceleration set are both zero before the gravity term is approximated, there are some limitations of the research. It is expected that the velocity observer can be added to the control algorithm proposed to broaden the depth of this research.

\section{References}

[1] Rybus T, Seweryn K, Sasiadek J Z. Control system for free-floating space mechanical arm based on nonlinear model predictive control (NMPC). Journal of Intelligent \& Robotic Systems, 2017, 85(3-4), pp. 491-509.

[2] Khan O, Pervaiz M, Ahmad E, et al. On the derivation of novel model and sophisticated control of flexible joint mechanical arm. Revue Roumaine des Sciences Techniques-Serie Electrotechnique et Energetique, 2017, 62(1), pp. 103-108.

[3] Liu Z, Liu J, He W. Dynamic modeling and vibration control for a nonlinear 3-dimensional flexible mechanical arm. International Journal of Robust and Nonlinear Control, 2018, 28(13), pp. 3927-3945.

[4] S. Sathishkumar, Dr. M. Kannan (2019). Topology Optimization of Integrated Combustion Engine Piston Using $\mathrm{F}$ ea Method (Cae Tools). Acta Mechanica Malaysia, 2(1): 0105.

[5] Iqbal J, Ullah M, Khan S G, et al. Nonlinear control systems-A brief overview of historical and recent advances. Nonlinear Engineering, 2017, 6(4), pp. 301-312.

[6] Xia K, Gao H, Ding L, et al. Trajectory tracking control of wheeled mobile mechanical arm based on fuzzy neural network and extended Kalman filtering. Neural Computing and Applications, 2018, 30(2), pp. 447-462.

[7] L. Natrayan, E. Aravindaraj, M.S. Santhosh, M. Senthil Kumar (2019). Analysis and Optimization of Connecting Tie Rod Assembly In Agriculture Application. Acta Mechanica Malaysia, 3(1): 06-10.

[8] Zhang Y, Yan P. Modeling, identification and compensation of hysteresis nonlinearity for a piezoelectric nano-mechanical arm. Journal of Intelligent Material Systems and Structures, 2017, 28(7), pp. 907-922.

[9] Farah Ruzanna Ridzuan, Hairulnizam Mahdin, Shahreen Kasim, Mohd Sanusi Azmi (2019). An Image-Based Captcha System Using Click. Acta Electronica Malaysia, 3(1): 23-25. 
[10] Wang H, Jiang N, Liu T, et al. Adaptive stable control of mechanical arm system based on immersion and invariance. Mathematical Modelling and Analysis, 2018, 23(3), pp. 379389.

[11] Ansarieshlaghi F, Eberhard P. Experimental study on a nonlinear observer application for a very flexible parallel robot. International Journal of Dynamics and Control, 2019, 7(3), pp. 1046-1055.

[12] Soh Hui Gian, Shahreen Kasim, Rohayanti Hassan, Zalmiyah Zakaria, Hairulnizam Mahdin, Azizul Azhar Ramli, Mohd Farhan Md Fudzee, Mohamad Aizi Salamat (2019). Online Activity Duration Management System for Manufacturing Company. Acta Electronica Malaysia, 3(2): 01-08.
[13] Morlock M, Burkhardt C S M, Seifried R. Nonlinear state estimation for trajectory tracking of a flexible parallel mechanical arm. IFAC-PapersOnLine, 2017, 50(1), pp. 34493454.

[14] Yang Z, Peng J, Liu Y. Adaptive neural network force tracking impedance control for uncertain robotic mechanical arm based on nonlinear velocity observer. Neurocomputing, 2019, 331, pp. 263-280.

[15] Muhammmad Ikram, Abbas Muhammad, Atiq Ur Rahmn (2019). Analytic Solution To Benjamin-Bona-Mahony Equation By Using Laplace Adomian Decomposition Method. Matrix Science Mathematic, 3(1): 01-04.

[16] Liu Z, Liu J, He W. Partial differential equation boundary control of a flexible mechanical arm with input saturation. International Journal of Systems Science, 2017, 48(1), pp. 53-62. 